\title{
Virtual Reality and Physical Activity: Attentional and Behavioral Aspects
}

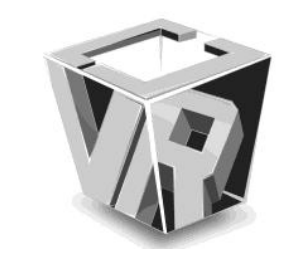

\author{
Virginie Dagonneau ${ }^{1,2}$, Christophe Maïano ${ }^{1}$, Charles-Symphorien Mercier $^{3}$ and Daniel R. Mestre ${ }^{1}$ \\ ${ }^{1}$ Institut des Sciences du Mouvement, CNRS \& Université de la Méditerranée, Marseille, France \\ ${ }^{2}$ Laboratoire Exploitation, Perception, Simulateurs et Simulations, INRETS-LCPC, Paris, France \\ ${ }^{3}$ Centre Médical Infantile Nutritionnel "Les Oiseaux”, Sanary-sur-mer, France.
}

\begin{abstract}
The present study tested the effects of sensorial inputs on performance and attentional focus during physical training on a computerized ergometer. 18 students were involved in this study. The experimental procedure consisted in a $3 \times 3 \times 4$ mixed design, with 3 practice modalities (virtual feedback, virtual feedback and music, virtual and heart rate feedback) 3 course phases (e.g., flat, uphill, downhill) and 4 sessions. The virtual feedback was a video film of the course subjects had to complete. Video display speed was proportional to the subject's pedalling speed. Results show a training effect on the subjects' overall performance and a reduction of the time spent gazing at video feedback across sessions. The results are discussed in terms of the functional status of sensorial stimulation and its interactions with exercise intensity and subject performance.
\end{abstract}

Index Terms-Attention, gaze behavior, Physical activity, Virtual reality

\section{INTRODUCTION}

The purpose of virtual reality (VR) is to enable someone to execute a sensorimotor and cognitive activity in an artificial world, which can be imaginary, symbolic, or a simulation of some parts of the real world [1]. To a certain extent, VR characteristics allow researchers to overcome limitations of classical assessment tools of sensorimotor and cognitive processing during the control of action. It also allows us to have rigorous experimental control and to test "ecological" situations, promoting multi-sensory experience, interactivity and immersion of users in a virtual world $[2,3]$.

Several physical activities practised indoor can be coupled to a VR system [4-8]. In such conditions, users carry out an aerobic physical activity on an ergometer, equipped with a variable resistance device modulating the exercise intensity. A VR software receives data about the subject's performance and controls in real time the sensorial and force feedback sent to the subject, as a function of his/her progression in a virtual environment. However, beside a general claim that exercise enjoyment and performance would benefit from VR coupling, few studies have systematically investigated cognitive and behavioral effects of VR. For instance, some authors [8] reported positive effects on actual exertion during a virtual reality-enhanced bicycling task, while others [6] found that behavioral effects seem to be dependant on informational format and content of a virtual environment.

One interesting way of thinking the links between cognitive processes and performance is suggested by Annesi [9]. He proposes that, during physical exercise, external sensorial stimulation (music and television in his study) might favour an external attentional focus. The idea is that an external attentional focus, diverting the subject from painful body sensations (related to exercise) would promote positive affects. On the contrary, an internal attentional focus would prevail when subjects' attention is focused on internal sensations [10, 11]. However, relationships between attentional focus and performance are not straightforward [12], seemingly depending on exercise intensity [13].

In this framework, we may ask whether a virtual reality system, coupling a subject's physical activity to his/her displacement inside a virtual environment would promote an internal or an external attentional focus. We can further suppose that this internal/external attentional balance will depend on the virtual feedback informational format and on exercise intensity. We can finally attempt to evaluate potential related effects of the virtual reality system on performance.

In order to do so, we designed an experimental study, in which three conditions of virtual reality feedback were tested, in a bicycling task performed on an ergometer:

In a first condition, we tested the effect of a virtual reality feedback, coupling the subject's pedalling speed to the display speed of a pre-recorded video of a real race. In a second condition, we added on the video alphanumerical information about the subject's instantaneous heart rate. This condition was supposed to favour an internal attentional focus. In a third condition, subjects could listen to music while watching the video. This last condition was supposed to favour an external attentional style.

Finally, we tried to assess attentional focus by analysing, the subjects' gaze orientation. Indeed, gaze orientation appears as a behavioral indicator of the allocation of attention in an environmental scene [14]. 


\section{METHODS}

\subsection{Participants}

A sample of 10 male and 8 female students aged between 21 and 25 years $(M=22.71, S D=12.31)$ took part in this study. Each participant was selected among the 8 following inclusion criteria : (i) age between 18 and 30 years, (ii) to be a man or a woman, (iii) to have no medical contraindication to practise physical activity, (iv) to be physically active (i.e. have a physical activity level over 3000 Standard Metabolic Equivalent), (v) to have a Body Max Index (BMI) between 18.5 and 24.9, (vi) have a measurement of waist under 88-90 $\mathrm{cm}$ for women and 100-102 $\mathrm{cm}$ for men, (vii) to have a waist/hips ratio strictly less than 0.85 for a women and 0.95 for a men and (viii) to have no previous experience in cycling practice in sports club. The different measures were obtained during an inclusion phase.

\subsection{Inclusion}

The inclusion phase was conducted during two sessions with one week interval in presence of a physician (third author). During the first session, participants completed a series of questionnaires exploring health state and physical activity level. During the second session, all participants had a personal interview with the same physician. Finally, participants performed a maximal exhausting test: Luc-Léger Test [15].

\subsection{Experiment}

\section{1) Apparatus}

The experimental setup consisted a fixed-base racing bike installed in front of a screen (Fig. 1). The bike was stationary on the floor and connected to a computer on which a RealAxiom (Elite ${ }^{\circledR}$ ) VR software was running. The software controls the effort feedback sent to subjects through a roll on which the rear wheel is fixed. This effort feedback is expressed through an electromagnetic brake, whose resistance strength is proportional to the instantaneous virtual course slope. The software also records subjects' pedalling speed, thanks to an optical detector placed on the same device (Fig. 2). Finally, the software uses the subject's pedalling speed to control the video display speed of the recorded course (visual feedback) on the screen in front of the subject. The display speed is proportional to the subject's pedalling speed. This video film (Fig. 3) displays a real course that was previously recorded by means of a camera installed onboard a car equipped with distance and altitude sensors. During the exercise, participants' face was recorded using a video camera, placed on the screen (Fig. 1) during visual feedback exercise, as well as heart rate (Polar electro S410, Finland).

\section{2) Procedure}

Participants were pseudo-randomly assigned to one of three experimental groups, according to gender, physical activity level and maximum oxygen uptake. Three inter-group experimental conditions were tested:

Group 1 was assigned to "Music and Virtual Reality

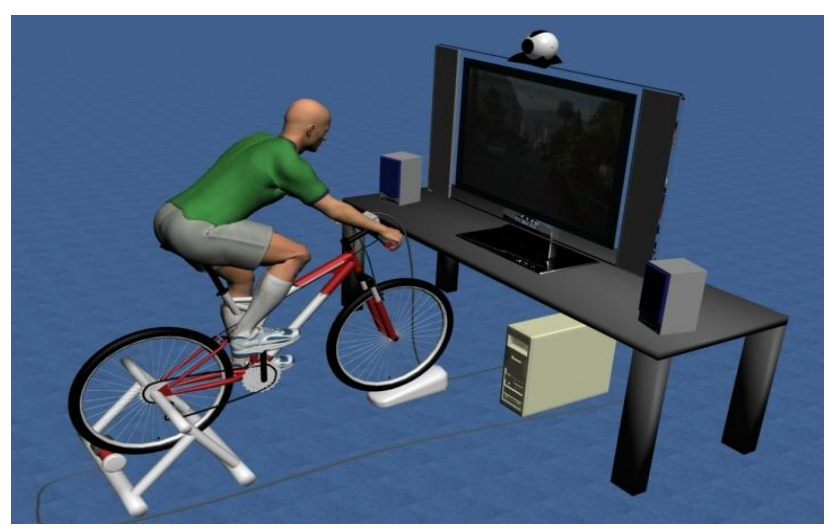

Fig. 1. Representation of the experimental setup.

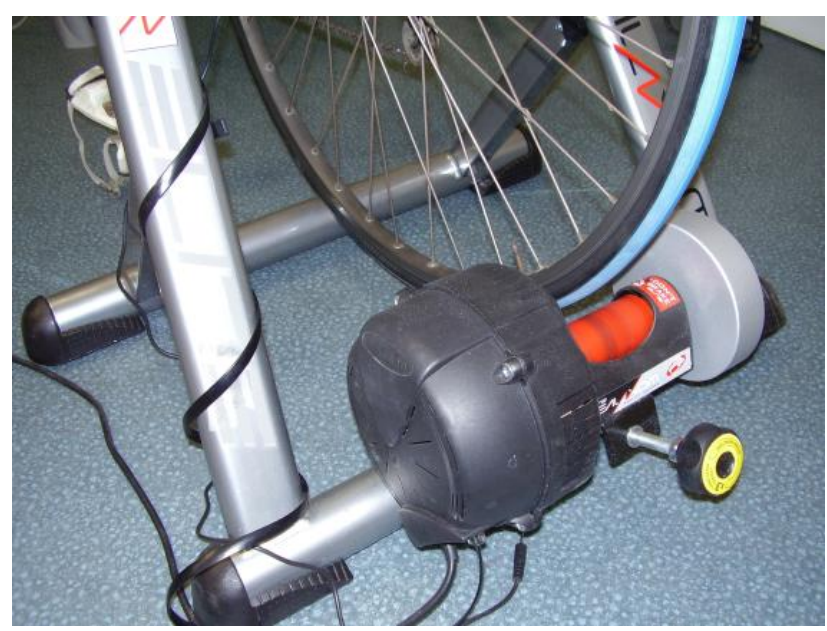

Fig. 2. Photo of the resistance tool (electromagnetic brake), in contact with the rear wheel.

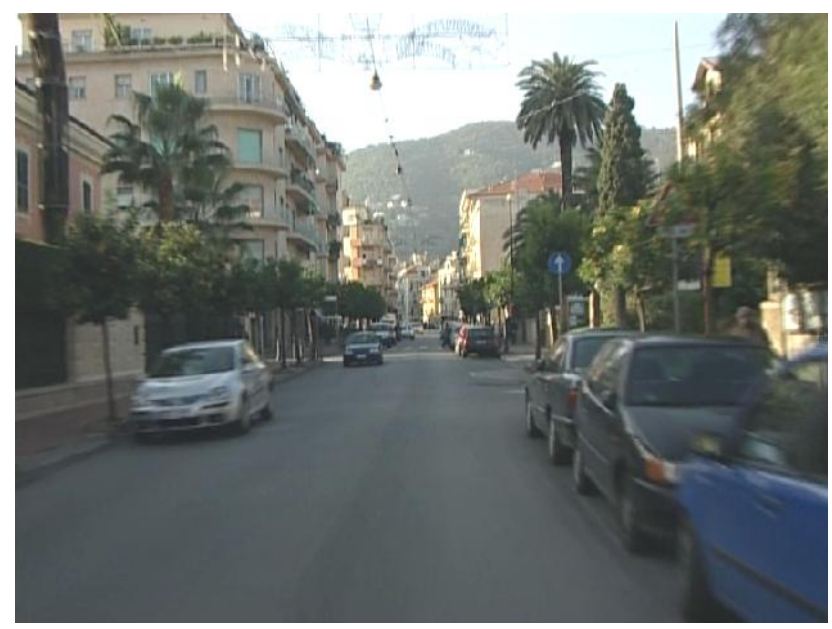

Fig. 3. Display of course video feedback (here departure of the course)

(Music-VR)" condition. In addition to being exposed to the virtual reality software stimulation, subjects had to wear earphones while exercising, in order to to listen to their favourite music during exercise. 
Group 2 was assigned to "Virtual Reality (VR)"condition (e.g., VR-controlled video feedback alone).

Group 3 was assigned to "Heart Rate and Virtual Reality (HR-VR)" condition. In this group, instantaneous heart rate was inserted on the display screen. However, no specific instruction was given to them, regarding this information.

Each subject completed 4 experimental sessions, with one week interval, on a fixed schedule.

For all groups, in order to define a baseline, the first session (T0) was performed in the absence of video feedback. The subsequent sessions (T1, T2, and T3) were performed on the same course with video feedback. Participants were asked to complete a course at their chosen speed, but without modifying gear ratio and gearwheel. Subjects had no feedback concerning exercise duration or remaining distance to the finish line, which was figured on the display screen. The exercise consisted in travelling a $7.5 \mathrm{~km}$ long course (real scenery), with a total change in altitude of $78 \mathrm{~m}$. This course (i.e., Fig. 4) was divided into 3 phases: (i) flat (from 0 to 3 $\mathrm{km}$ ), (ii) uphill (from 3.1 to $4.8 \mathrm{~km}$ ), (iii) downhill (from 4.81 to $7.5 \mathrm{~km})$.

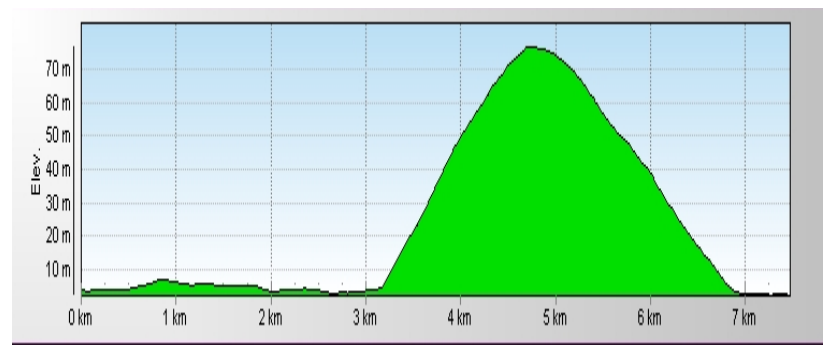

Fig. 4. Characteristic of the course topology, with three successive phases: flat, from 0 to $3 \mathrm{~km}$, uphill between 3 and about $5 \mathrm{~km}$ and downhill.

\section{3) Data recording and analysis}

Heart rate (expressed in bpm) and power (in watts) were recorded during the exercise by the VR software (@1 Hz) and stored for off-line analysis. Gaze behavior was independently recorded on a video file. It was manually synchronized to the preceding data.

From these data, mean heart rate and mean power were calculated for each course phase. From the "gaze" video, gaze time was defined as a ratio between the amount of time spent looking at the screen and the total exercise time.

\section{4) Statistical analysis}

Three-way ANOVAS were applied to these data, with Time (4 sessions: T0 to T4) and Phase (3 course phases: flat, uphill, downhill) as intra-group factors and Modality (3 modalities: Music-VR, VR, HR-VR) as between-group factor.

\section{RESULTS}

\subsection{Heart rate}

A significant difference is observed between course phases $[F(2,28)=106.71, p<0.001]$. Post-hoc analysis indicates that mean heart rate was significantly higher in uphill phase (161 $\mathrm{bpm} \pm 15 \mathrm{bpm})$ than in flat phase (125 bpm $\pm 17 \mathrm{bpm})$ and downhill phase (147 bpm \pm 24$)$, and also higher in downhill phase than in flat phase. Moreover, an interaction effect between Time and Modality is observed $[\mathrm{F}(6,42)=2.29$, $\mathrm{p}=0.05]$. For "Music-VR" modality a significant increase in average heart rate is observed between times T0-T2 and T0-T3. For "VR" modality a significant decrease exists between times T0-T1 and T0-T2. No significant effect of Time factor is observed for "HR-VR" modality (fig. 5).

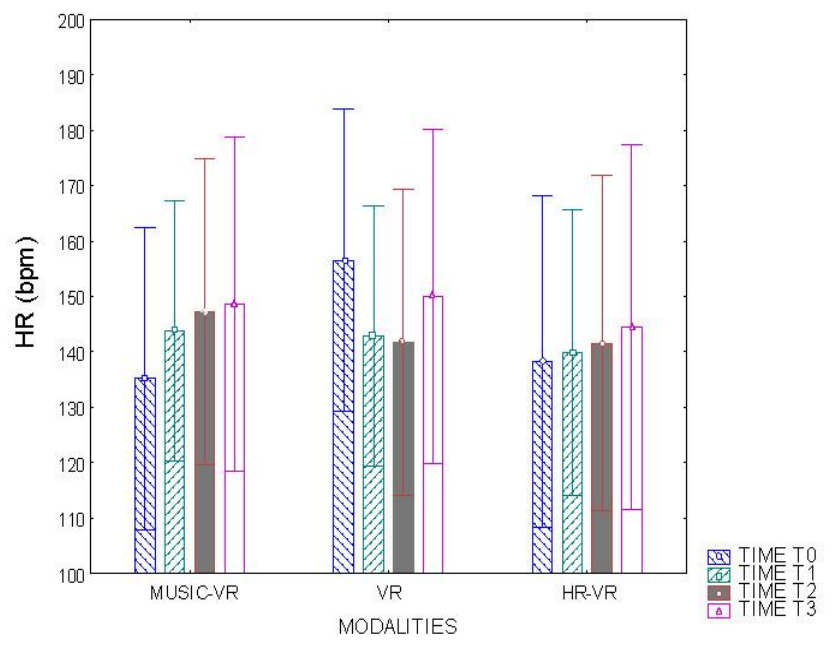

Fig. 5. Interaction effects between Time and Modality factors on mean heart rate

\subsection{Power}

A significant Time effect is observed $[F(3,42)=3.49$, $\mathrm{p}<0.05]$. Post-Hoc Fisher LSD test specifies that mean power is significantly higher in $\mathrm{T} 3(124.21 \mathrm{~W} \pm 32.74 \mathrm{~W})$, as compared to $\mathrm{T} 0(111.69 \mathrm{~W} \pm 67.77 \mathrm{~W})$ and $\mathrm{T} 1(109.72 \mathrm{~W} \pm$ $24.62 \mathrm{~W})$. A significant Phase effect $[\mathrm{F}(2,28)=145.96$, $\mathrm{p}<0.05]$ is also noticed. Fisher LSD test indicates that mean power is higher in uphill phase $(185.61 \mathrm{~W} \pm 4.47 \mathrm{~W})$ than in flat phase $(108.37 \mathrm{~W} \pm 9.44 \mathrm{~W})$ and downhill phase (45.71 $\mathrm{W} \pm 5.65 \mathrm{~W})$.

A significant interaction effect between Phase and Time factors is observed $[\mathrm{F}(6,84)=3.00, \mathrm{p}<0.05]$. Post-hoc analysis indicates that (i) in flat phase, mean power is lower in $\mathrm{T} 1(96.05 \mathrm{~W} \pm 19.53 \mathrm{~W})$ than in $\mathrm{T} 0(111.25 \mathrm{~W} \pm 39.87 \mathrm{~W})$ and $\mathrm{T} 2(107.49 \mathrm{~W} \pm 30.17 \mathrm{~W})$ and $\mathrm{T} 3(118.70 \mathrm{~W} \pm 38.39 \mathrm{~W})$ and that mean power is also higher in T3 than in T2; (ii) in uphill phase, no significant difference between Times is noticed.; (iii) in downhill phase, mean power is higher in T3 $(52.02 \mathrm{~W} \pm 24.89 \mathrm{~W})$ than in $\mathrm{T} 2(48.28 \mathrm{~W} \pm 21.23 \mathrm{~W}), \mathrm{T} 1$ $(39.04 \mathrm{~W} \pm 16.57 \mathrm{~W})$ and $\mathrm{T} 0(43.51 \mathrm{~W} \pm 19.60 \mathrm{~W})$.

\subsection{Gaze time}

Gaze time analysis was only performed during visual feedback sessions (i.e. T1-T2-T3).

The three-way analysis of variance indicates a significant effect of Time $[F(2,28)=6.97, \mathrm{p}<0.01]$, Phase $[F(2,28)=7.43$, $\mathrm{p}<0.01]$, and an interaction effect between Phase and 
Modality $[\mathrm{F}(4,28)=3.28, \mathrm{p}<0.05]$.

Concerning the Time effect, Fisher LSD test indicates that gaze time was higher in $\mathrm{T} 1(77 \% \pm 22 \%)$ than in $\mathrm{T} 2(64 \% \pm$ $27 \%)$ and $\mathrm{T} 3(59 \% \pm 27)$.

Concerning the Phase effect, post-hoc analysis indicates a significant gaze time decrease when exercise intensity increases, between flat $(69 \% \pm 24 \%)$ and uphill phases $(60 \%$ $\pm 28 \%$ ) and inversely, a gaze time increase when exercise intensity decreases between uphill and downhill phases $(71 \%$ $\pm 26 \%$ ).

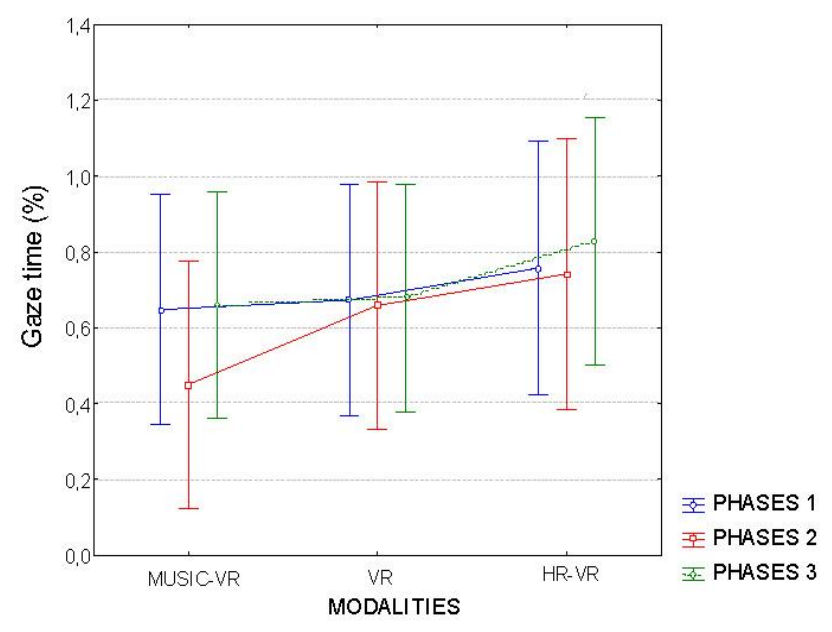

Fig. 6. Interaction effects between Phase and Modality on the percentage of time spent gazing at the screen

As regards the interaction effect between Phase and Modality, Fisher LSD test indicates, for "Music-VR" modality, that gaze time (i) is significantly higher in flat $(65 \% \pm 24)$ than in uphill phase $(45 \% \pm 21)$, and (ii) is higher in downhill $(66 \% \pm 27)$ than in uphill phase. However, no significant gaze time difference is observed for "VR" or "HR-VR" modalities (Fig. 6).

\section{DISCUSSION}

The purpose of this study was to experimentally manipulate sensorial inputs to a subject exercising on an ergometer, in order to evaluate their effect during a cycling task on a computer-controlled ergometer. Moreover, the experiment was specifically designed to study task repetition and task intensity effects.

Performance. First, as could be intuitively expected, results show a global intensity effect on performance, in particular heart rate and power indicators. This effect might be explained by a "mechanical" effect (simulation of the slope of the road with a resistance force applied to the rear bicycle wheel). Results also indicate a global effect of task repetition. In agreement with previous reports [16], our results suggest a learning effect during exercise in VR. Results also highlight an interaction effect between repetition and intensity, for the power parameter. The pattern of results suggests that the repetition effect only for the phases with low-to-moderate intensity.
Globally, the present results do not indicate any simple effect of the Modality factor (i.e., "Music and Virtual Reality, Virtual Reality, Heart Rate and Virtual Reality) in relation to T0 (i.e., without video feedback) and between "video" sessions (from $\mathrm{T} 1$ to $\mathrm{T} 3$ ). However, interactions exist between Modality and task repetition (Time factor). In particular, for the "Music-VR" condition, we notice an increase in average heart rate value with exercise repetition. On the opposite, for the group with a heart rate feedback (i.e., "HR-VR"), no significant difference in heart rate can be noticed. This result might suggest that this group keeps a stable heart rate over sessions. In agreement with a previous study [17], we can suggest that displaying heart rate allows subjects to manage their effort (homeostasis biofeedback loop). On the other hand, only the group with musical stimulation (i.e., "Music-VR") presents a significant decrease of the total exercise duration with task repetition. This might explain why these subjects present an elevation of mean heart rate with task repetition. This result suggests that music, distracting participants from the exercise [9], might favour performance.

Gaze behavior. Across all experimental conditions, subjects spend more than half of the exercise time (66\%) looking at the visual feedback.

However, gaze time (of visual feedback) decreases over sessions for all groups. This might be explained by the fact that the course (and the video film) was identical across sessions, such that, when a novelty effect had passed and/or learning of the course occurred, gaze attraction to the video feedback decreased accordingly. We can also suggest a differentiated use of the video feedback over sessions. Subjects might use visual feedback to estimate their progress (position) in the course. Another assumption, which has to be tested by a more detailed analysis of gaze time, is that the visual feedback (over sessions) allows them to anticipate, for example the end of the uphill phase.

Finally, results show a phase effect on the time spent gazing at video feedback only for "Music-VR" modality. Subjects listening to music during exercise are less attentive to video feedback, essentially when exercise intensity is higher (i.e., in uphill phase). On the contrary, subjects of "VR" and "HR-VR" groups continue to look at video feedback while exercise intensity increases. These results suggest, for "Music-VR" group, a different use of video feedback as compared to the other groups.

If we compare gaze behavior and performance results, we observe decrease of gaze time over sessions, but an increase of performance. Moreover, for subjects with music and visual feedback, when exercise intensity increases, they spend less time looking at the display screen, while increasing their power. This is not the case for the other two groups. These results address the problem of the functional status of video feedback. It is possible that some learning of the video itself occurred, explaining this decrease of the time spent gazing at video feedback. It is also possible that information seeking is influenced by exercise intensity and/or by the nature of sensorial stimulation. In addition, we can wonder if the use of information varies depending on subjects' practice level (i.e., 
novice, occasional, elite).

A more precise gaze analysis (i.e., using an eyetracker) seems appropriate to clarify this point. Indeed, gaze analysis used in this study did not allow us to distinguish gazing strategies of subjects (i.e., participants looking at the video feedback for brief durations, as compared to participants looking continually at the video scene), and did not allow to distinguish gazing zones in the visual scene.

\section{CONCLUSIONS}

In relation with previous studies suggesting a positive role of interactive virtual environments on physical activity, our results report complex interactions between exercise intensity, sensorial information and performance. Sensorial information can be distinguished between "external" information (i.e., not linked to exercise, for example music but also landscape in the visual scene) and "internal" information, linked to exercise. This latter can also be separated into "symbolic" (i.e., duration, distance, speed, heart rate) and "ecological" (optic flow), information. In order to achieve better understanding of these interactions, future experimental studies should systematically manipulate the status of visual information. It appears relevant to study a continuum starting from a stimulation disconnected from the subject's activity (i.e., film) to an interactive visual stimulation in which the subject could control not only his/her displacement speed (as in our work) but also his trajectory. We can also manipulate the complexity of the virtual environment (i.e., from an abstract optic flow to a complex scenery), so as to test the distracting status of visual information.

This work suggests the interest of gaze direction analysis as a tool to study attentional processes linked to physical practice. This method needs to be developed, in particular with more precise apparatus, allowing us to estimate gaze strategies (fixation locations, fixation times). In addition, considering the attentional focus, we attempted to influence it by a manipulation of experimental conditions with minimal instructions given to the subjects (i.e., to complete the track). Attentional focus having inevitably a subjective aspect, a controlled manipulation of instructions might be tested. In particular, we suggest to test, in conjunction with the manipulation of sensorial information, instruction effects aiming at favoring a focus of subjects' attention on their movements (e.g., to keep a regular cadency) or on the movement effect (e.g., to maintain a constant distance with an opponent) [18].

Finally, our study made a modest contribution to understanding functional links between strategies to acquire visual information, attentional processes and performance. In particular, we noticed significant relationships between gaze direction and power, when exercise conditions were relatively intense. This first result has to be confirmed. It will require to study more precisely physiological conditions of power manifestations, according to exercise intensity, and to overcome an average measurement of power. To conclude, this work suggests the interest of VR tool to study the complex links between strategies used to acquire information and physical performance.

\section{REFERENCES}

[1] P. Fuchs, G. Moreau, A. Berthoz and J. L Vercher. Le traité de la réalité virtuelle - Volume 1 L'homme et l'environnement virtuel. Presses de l'Ecole des Mines de Paris, Paris, 2006.

[2] J. M. Loomis, J. J. Blascovich and A. C. Beall. Immersive virtual environment technology as a basic research tool in psychology, Behav Res Meth Instrum Comput, vol. 31, no. 4, pp. 557-564, 1999.

[3] D. R. Mestre. Interactions entre Réalité Virtuelle et Sciences du Comportement. In P. Fuchs, G. Moreau, A. Berthoz and J.L. Vercher (Eds.), Traité de la Réalité Virtuelle, Volume 1: L'homme et l'environnement virtuel, Presses de l'Ecole des Mines de Paris, Paris, pp. 363-380, 2006.

[4] J. J. Annesiand J. Mazas. Effects of virtual reality-enhanced exercise equipment on adherence and exercise-induced feeling states, Percep Mot Skills, vol. 85, no. 3, pp. 835-844, 1997.

[5] T.-Y. Chuang, C.-H. Chen, H.-A. Chang, H.-C. Lee, C.-L. Chouand J.-L. Doong. Virtual reality serves as a support technology in cardiopulmonary exercise testing, Presence, vol. 12, no. 3, pp. 326-331, 2003.

[6] W. A. Ijsselsteijn, Y. A. De Kort, J. Westerink, M. De Jaggerand R. Bonants. Virtual Fitness: Stimulating exercise behavior through media technology, Presence, vol. 15, no. 6, pp. 688-698, 2006.

[7] H. S.-H. Mac Rae, C. L. Miller-Perrinand C. M. Tinberg. Cycling with video feedback improves performance in untrained, but not in trained women, Res Sports Med, vol. 11, no. 4, pp. 261-276, 2003.

[8] T. G. Plante, A. Aldridge, R. Bogden and C. Hanelin. Might virtual reality promote the mood benefits of exercise? Comput Human Behav, vol. 19 , no. 4 , pp. 495-509, 2003.

[9] J. J. Annesi. Effects of music, television, and a combination entertainment system on distraction, exercice adherence, and physical output in adults, Can J Behav Sci, vol. 33, no. 3, pp. 193-202, 2001.

[10] W. P. Morgan and M. L. Pollock. Psychologic characterization of the elite distance runner, Ann N Y Acad Sci, vol. 301, pp. 382-403, 1977.

[11] K. S. Master and B. M. Ogles. Associative dissociative cognitives strategies in exercise and running: 20 years later, What do we know? Sport Psychol, vol. 12, no. 3, pp. 253-270, 1998.

[12] W. D Russell and D. L. Weeks. Attentional style in ratings of perceived exertion during physical exercise, Percept Mot Skills, vol. 78, no. 3, pp. 779-783, 1994.

[13] J. C. Hutchinsonand G. Tenenbaum. Attention focus during physical effort: The mediating role of task intensity, Psychol Sport Exerc, vol. 8, no. 2, pp. 233-245, 2007.

[14] J. M. Henderson. Human gaze control during real-world scene perception, Trends Cogn Sci, vol. 7, no. 11, pp. 498-504, 2003.

[15] L. Léger and J. Lambert. A maximal multistage 20-m shuttle run test to predict $\mathrm{VO}_{2}$ max, Eur JAppl Physiol, vol. 49, no. 1, pp. 1-12, 1982.

[16] M. L. Scott, D. Scott, S. P. Bedic and J. Dowd. The effect of associative and dissociative strategies on rowing ergometer performance. Sport Psychol, vol. 13, no. 1, pp 57-68, 1999.

[17] A. St Clair Gibson, E. V. Lambert, L. H. G. Rauch, R. Tucker, D. A. Baden, C. Foster and T. D Noakes. The role of information processing between the brain and peripheral physiological systems in pacing and perception of effort. Sports Med, vol. 36, no. 8, pp. 706-722, 2006.

[18] G. Wulf., M. Hoss and W. Prinz. Instructions for motor learning: Differential effects of internal versus external focus of attention. J Mot Behav, vol. 30, no. 2, pp. 169-179, 1998.

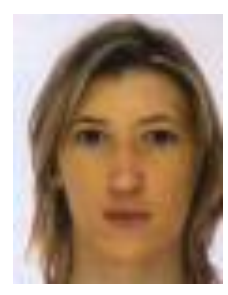

Virginie Dagonneau is currently a PhD Student studying Human Behavioral Science at the Laboratory for road operation, perception, simulations and simulators (LEPSIS), in Paris (France). Her $\mathrm{PhD}$ focuses on the links between immersion and presence to develop a powered two-wheelers simulator.

During her Master studies at the Sports Sciences Faculty (Marseille), she conducted, in the 
Immersions Group of the Institute of Movement Sciences, experimental investigations of the positive role of Virtual Reality feedback during physical exercise.

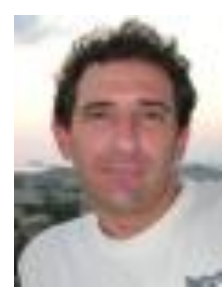

Christophe Maïano is a lecturer at the Faculty of Sports Sciences, University of Aix-Marseille II (http://www.staps.univ-mrs.fr/) and researcher in the Institute of Movement Sciences (ISM). His research interests are fourfold: (i) virtual reality, health and physical activity; (ii) social psychology, health and physical activity; (iii) psychometrics, health and physical activity; and (iv) virtual reality, anxiety and human behavior. Since 2006 and his recruitment at the ISM he has been working on (i) how to use virtual reality and physical activity in rehabilitation research, and (ii) how anxiety could have an influence on movement behavior in virtual reality environments. He is currently studying how virtual reality home-trainers could be used to improve the rehabilitation of obese adolescents.

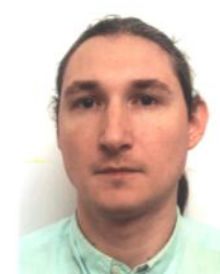

Charles-Symphorien Mercier is a physicist, specialized in nutrition in infantile obesity. He is a clinical investigator in "Les Oiseaux" Nutritional Medical Center, Sanary/Mer. He is currently pursuing a $\mathrm{PhD}$ in the field of medical sciences. (Medicine Faculty, Marseille). He is a collaborator of the Sports Sciences Faculty in Marseille and coordinates experimental studies in the Immersions Group (Institute of Movement Sciences).

Since 2006, his research specialty consists in identifying predictive factors of the therapeutic response to a multidisciplinary intervention toward obese teenagers

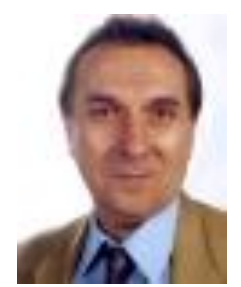

Daniel R. Mestre is a senior researcher in the Institute of Movement Sciences (www.ism. univmed.fr) in Marseilles. He is Director of the Immersions group and Head of the Mediterranean Virtual Reality Center (www.realite-virtuelle. univmed.fr).

His major research interests concern visual motion perception, psychophysical and neuropsychological approaches to sensory information processing and virtual reality as an experimental tool for sensori-motor interactions, in both fundamental and applied domains 